Tohoku J. Exp. Med., 2010, 221, 43-48

\title{
Ergometry Induces Systemic Oxidative Stress in Healthy Human Subjects
}

\author{
Maja Antoncic-Svetina, ${ }^{1}$ Davor Sentija, ${ }^{2}$ Ana Cipak,${ }^{3}$ Davor Milicic, ${ }^{4}$ \\ Andreas Meinitzer, ${ }^{5}$ Franz Tatzber, ${ }^{6}$ Luka Andrisic, ${ }^{3}$ Sieglinde Zelzer ${ }^{5}$ and \\ Neven Zarkovic ${ }^{3}$

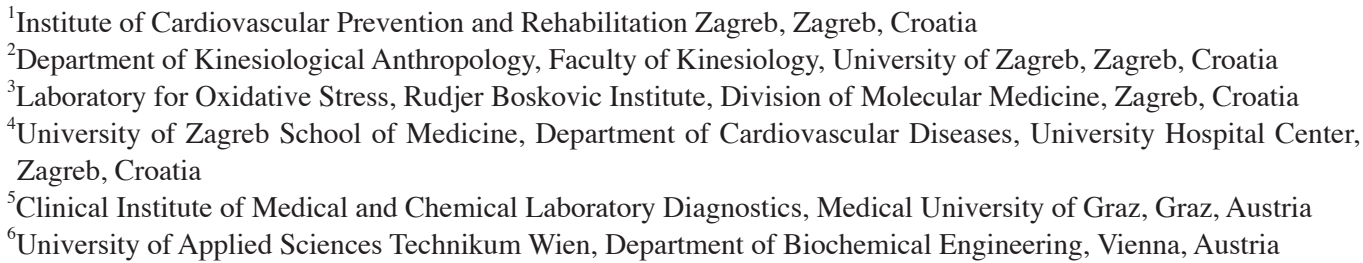

Oxidative stress is an important pathogenic factor of cancer and cardiovascular, metabolic and degenerative diseases. On the other hand, mild oxidative stress, as in case of physical exercise, can increase the antioxidant defense system. However, the mechanisms underlying such desirable effects of mild oxidative stress are not well understood, because the production of hydroxyl radical, the most aggressive oxygen free radical, was not yet evaluated under physiological circumstances. Therefore, in this study, we evaluated the overall production of hydroxyl radical using blood samples of ten healthy male students before and $1 \mathrm{~h}$ after ergometry. One $\mathrm{h}$ before exercise, they took salicylic acid $(1 \mathrm{~g})$ orally so that hydroxyl radical was trapped with salicylic acid, yielding a measurable reaction product, 2,3dihydroxybenzoic acid. Oxidative stress response to exercise was also evaluated in the volunteers without premedication by measuring serum peroxides and total antioxidant capacity of serum. These parameters of oxidative stress were then correlated with physical performance of the subjects. Ergometry caused an increase of the plasma hydroxyl radical level by $37.5 \%(p<0.05)$, whereas the levels of total serum peroxides did not change significantly. Total serum antioxidant capacity, measured as uric acid equivalents, was higher after ergometry by $39.7 \%(p<0.05)$, and was in positive correlation $(r=0.81)$ with anaerobic threshold, an indicator of physical condition. Hence, ergometry induces hydroxyl radical production and systemic oxidative stress response in the healthy subjects. Egometry could be used to study physiological oxidative stress response and to improve antioxidant defense capacities in humans.

Keywords: hydroxyl radical; serum peroxides; antioxidants; oxidative homeostasis; ergometry

Tohoku J. Exp. Med., 2010, 221 (1), 43-48. (C) 2010 Tohoku University Medical Press

Excess in production of oxygen free radicals can damage the important molecules, nucleic acids, proteins, lipids and carbohydrates in a process denoted oxidative stress. These complex macromolecules are affected by free radicals because free radicals have "unpaired" electrons and are consequently extremely unstable, so they rapidly react with macromolecules to stabilize the electron/atom structure. These interactions between free radicals and macromolecules affect structure and function of macromolecules and might even result in their disintegration. Therefore, oxidative stress is an important pathogenic factor of ageing and various disorders, such as cancer, autoimmune, cardiovascular, metabolic and neurodegenerative diseases (Isik et al. 2007; Mamiya et al. 2008; Ates et al. 2009; Tasoulis et al. 2009). On the other hand, mild and reversible oxidative stress, as in case of physical exercise, can increase the defense against oxidative stress. Such a defense is based on the activities of enzymatic and non-enzymatic antioxidants, which react with free radicals to stabilize them and prevent damage to the macromolecules, but the mechanisms underlying the desirable effects of mild oxidative stress are not well understood.

To attenuate the damage caused by oxidative stress and to avoid interaction of reactive oxygen substances/species (ROS) with macromolecules, cells have evolved different detoxification mechanisms (Jamieson 1998; Urso and Clarkson 2003). Non-enzymatic mechanisms involve small hydrophobic or hydrophilic molecules that act as radical scavengers, such as glutathione, vitamin $\mathrm{C}$, vitamin $\mathrm{E}$ and similar antioxidants (Ji et al. 2008). Enzymatic mechanisms

Received January 28, 2010; revision accepted for publication March 23, 2010. doi:10.1620/tjem.221.43

Correspondence: Neven Zarkovic, M.D., Ph.D., Senior Scientist, Rudjer Boskovic Institute, Bijenicka 54, HR-1000 Zagreb, Croatia.

e-mail: zarkovic@irb.hr 
for ROS detoxification are more complex and consist of enzymatic cascades, among which the most prominent cascades include superoxide dismutase (SOD) and catalase (Jamieson 1998; Ji et al. 2008). Therefore, measurement of total antioxidant capacity is one of the convenient parameters reflecting oxidative stress and homeostasis and is complementary to the measurement of the ROS, such as measurement of the total peroxide levels.

Exercise-induced oxidative stress became an attractive research topic in recent years, since it seems to be fundamental for hormesis and consequently, oxidative homeostasis (Bloomer 2008; Wildburger et al. 2009). Namely, the concepts of hormesis and oxidative homeostasis consider mild oxidative stress as stimulant for development of antioxidant defense system maintaining the steady state of proand anti-oxidants (oxidative homeostasis), which might consequently result in more efficient defense of the organism against severe oxidative stress (hormesis).

In favor of this concept, exercise of sufficient duration and intensity may lead to formation of oxygen free radicals and other ROS (Bloomer 2008). It is commonly accepted that only exhaustive exercise generates systemic oxidative stress response (Gomez-Cabrera et al. 2008). Nonexhaustive exercise results in too mild increase of ROS production, which can be rapidly attenuated by endogenous antioxidant defense and therefore does not cause systemic oxidative stress response (Vina et al. 2000; Cindric et al. 2009). During exercise, main source of ROS in skeletal muscle might be mitochondrial oxidative metabolism necessary to provide the energy for the physical, in particular muscular, activity (Bejma and Ji 1999). In addition, the enzyme xantine oxidase, located in the vascular endothelial cells in skeletal muscle (Hellsten-Westing 1993; GomezCabrera et al. 2006) together with transition metals in the cytoplasm of the cells, e.g. iron, generate ROS, including the most aggressive hydroxyl radical $\left({ }^{\circ} \mathrm{OH}\right)$. This radical is the most harmful ROS, and is formed in excess only during severe oxidative stress and seems to be the only radical able to initiate lipid peroxidation (Braughler and Hall 1989; Halliwell 1996). Because lipids are essential elements of the cellular membranes, oxidative stress generating ${ }^{\circ} \mathrm{OH}$ results in lipid peroxidation, which may alter structure and function of the biomembranes. Accordingly, lipid peroxidation and its end products, reactive aldehydes, denoted as second messengers of free radicals, are implicated in pathogenesis of various diseases, associated with oxidative stress (Esterbauer et al. 1991; Zarkovic 2003; Wildburger at al. 2009). Among these aldehydes, malondialdehyde (MDA), 4-hydroxynonenal (HNE) and acrolein are the most investigated. Thus, recent findings of the physiological presence of these aldehydes in various normal and healthy human and animal tissues indicate that antioxidant defense systems can maintain oxidative homeostasis even after lipid peroxidation occurred (Zarkovic 2009). However, we lack evidence that even mild, physiological oxidative stress, as in case of physical exercise, can cause systemic oxidative stress response based on excessive generation of ${ }^{\circ} \mathrm{OH}$ in healthy human subjects. Thus, the proof of excessive generation of ${ }^{\circ} \mathrm{OH}$ in healthy humans, developed under physiological, reversible circumstances, such as exercise, could provide novel insight into the fundamental aspects of oxidative homeostasis. This may open doors for the use of ergometry to study physiological systemic oxidative stress response and to promote physical exercise as a simple method for enhancing the antioxidant defense capacities of the organism.

Therefore, we determined ${ }^{\circ} \mathrm{OH}$ production using the blood samples of healthy volunteers after exercise and the oxidative stress response to ergometry reflected by the change of total serum peroxides and total antioxidant capacity of serum.

\section{Materials and Methods}

\section{Subjects}

Ten healthy male physical education students, who all regularly every day practice moderate exercise, participated in the study $(21 \pm 1$ years old). The volunteers did not receive any medication, except for the experiment, in which ${ }^{\circ} \mathrm{OH}$ trapping was performed with the use of acetyl-salicylic acid $(1 \mathrm{~g})$ orally one hour before the exercise. In case of analogous experiment done for the determination of the serum peroxide levels and total antioxidant capacity, they did not receive any premedication. The measurement procedures and potential risks were explained to each volunteer, so the written informed consents were collected, in accordance with guidelines of the Helsinki Declaration.

\section{Testing procedure}

Subjects: All subjects were familiarized with treadmill walking and running for a period of at least 15 min prior to their first data collection. Both tests were performed on a motorized, calibrated treadmill with speed resolution of $0.1 \mathrm{~km} \cdot \mathrm{h}^{-1}$ (Run Race Technogym, Gambettola, Italy), and preceded by a short warm-up and stretching procedure. Gas exchange data were measured breath-by-breath using a metabolic measurement cart (Quark $b^{2}$, Cosmed, Italy). Before each test, the gas analyzers were calibrated using gases of known concentration, and the flow meter was calibrated using a 3-L syringe. Heart rate (HR) was recorded during the tests using a HR monitor (Polar Electro, Kempele, Finland). The two testing sessions were separated by $2-4$ days, and performed in randomized order.

Maximal running test $\left(V_{2 \max }\right.$ test): The starting speed of 3 $\mathrm{km} \cdot \mathrm{h}^{-1}$ was maintained for $3 \mathrm{~min}$, after which the speed was increased by $0.5 \mathrm{~km} \cdot \mathrm{h}^{-1}$ every $30 \mathrm{sec}$. The subjects walked the first stages (up to $6.5 \mathrm{~km} \cdot \mathrm{h}^{-1}$ ), and continued running from $7 \mathrm{~km} \cdot \mathrm{h}^{-1}$ until volitional exhaustion. The last full stage the subject could sustain (for $30 \mathrm{sec}$ ) was defined as the subject's maximal speed. During recovery, the subjects walked at $5 \mathrm{~km} \cdot \mathrm{h}^{-1}$ for $5 \mathrm{~min}$.

Data collection and analysis: After completion of the tests, gas exchange data and HR were averaged at 30 -sec intervals. Graphical determination of the anaerobic gas exchange threshold for running (anaerobic threshold, equivalent to the maximal lactate steady state) was performed using the simplified V-slope method according to the previously published recommendation (Meyer et al. 2005). Briefly, the V-slope method allows detection of both, aerobic and anaerobic 
Table 1. Physical performance parameters of the subjects on a motorized, calibrated treadmill.

\begin{tabular}{lc}
\hline $\mathrm{VO}_{2 \max }(\mathrm{L} / \mathrm{min})$ & $4.65 \pm 0.21$ \\
$\mathrm{VO}_{2 \max } / \mathrm{kg}(\mathrm{ml} / \mathrm{min} / \mathrm{kg})$ & $58.2 \pm 4.4$ \\
$\mathrm{Max}$ heart rate $(\mathrm{bpm})$ & $192 \pm 9$ \\
$\mathrm{Max}$ ventilation $(\mathrm{L} / \mathrm{min})$ & $166 \pm 11$ \\
Max ventilatory equivalent & $35 \pm 2$ \\
Max running speed $(\mathrm{km} / \mathrm{h})$ & $17.5 \pm 1.34$ \\
Running speed at the anaerobic threshold $(\mathrm{km} / \mathrm{h})$ & $13.7 \pm 0.8$ \\
$\% \mathrm{VO}_{2 \max }$ at AT & $86 \pm 4$ \\
Heart rate at AT $(\mathrm{bpm})$ & $176 \pm 12$ \\
\hline
\end{tabular}

thresholds, based on accelerated rate of $\mathrm{CO}_{2}$ output compared to oxygen uptake. The running speed corresponding to the point of a second disproportionate increase in $\mathrm{VCO}_{2}$ in relation to $\mathrm{VO}_{2}$ was considered as the speed at the anaerobic threshold (AT). The AT is the turning point of physical exercise when oxygen is not provided sufficiently for the increased body demands to allow regular aerobic energy metabolism; thus, anaerobic metabolism begins to dominate, resulting in increase in lactate production that causes fatigue. When indeterminate, the V-slope method was supported with inspection of the respiratory exchange ratio $(\mathrm{R} \sim 1.00$ at $\mathrm{AT})$, ventilation and ventilatory equivalents for $\mathrm{O}_{2}$ and $\mathrm{CO}_{2}$. The highest oxygen uptake for any 30 -sec period recorded in the incremental running test was defined as $\mathrm{VO}_{2 \max }$. The data obtained from tests are shown in Table 1.

\section{Blood sampling}

Peripheral venous blood samples were collected from the forearm anterobrachial vein before the exercise and $60 \mathrm{~min}$ after the exercise. Blood $(10 \mathrm{ml})$ was taken in plastic tubes with EDTA and was centrifuged at $3000 \mathrm{rpm}$ for $15 \mathrm{~min}$. The plasma samples thus obtained were stored at $-80^{\circ} \mathrm{C}$ until analysis of ${ }^{\circ} \mathrm{OH}$ trapping by salicylic acid. In parallel serum samples were prepared and equally stored for the analyses of total serum antioxidant capacity and serum peroxide levels by rapid enzymatic in-vitro assays.

\section{Analysis of hydroxyl radical ( $\left.{ }^{\circ} \mathrm{OH}\right)$ in plasma}

For measurement of hydroxyl radical ${ }^{\circ} \mathrm{OH}$, indirect methods are used to determine aromatic acids hydroxylation products. Salicylic acid is most commonly used, since its hydroxylation products 2,3dihydroxybenzoic acid (2,3-DHBA) and 2,5-dihydroxybenzoic acid (2,5-DHBA) can be separated and quantified by high performance liquid chromatography (HPLC) with electrochemical detection (McCabe et al. 1997). As 2,5-DHBA can be produced also by enzymatic pathway, only 2,3-DHBA serves as the most reliable method for determination of ${ }^{\circ} \mathrm{OH}$ radical production (Borovic et al. 2000; Khoschsorur et al. 2000).

For DHBA determination, samples were extracted with hydrochloric acid and diethylether with 3,4-DHBA as internal standard. Separation was performed on $150 \times 4.6$ Waters Spherisorb ODS2 $3-\mu \mathrm{m}$ column with $7.48 \mathrm{mM}$ sodium citrate/acetic acid mobile phase pH 4.6 and $3 \%$ methanol. Products were detected with Shimadzu LC-20A Prominence (Shimadzu, Kyoto, Japan) with electrochemical detector set on $400 \mathrm{mV}$ and flow on $0.6 \mathrm{ml} / \mathrm{min}$.

\section{Total antioxidant capacity}

The total antioxidant capacity of serum was determined as described before by a commercially available assay (TAC LND, Germany) (Nikolic-Heitzler et al. 2006). The assay is based on serum antioxidant consumption after addition of hydrogen peroxide. Serial dilutions of uric acid, one of the most potent serum antioxidant molecules, were used for standardization. Results were expressed as equivalents of uric acid (in $\mathrm{mM}$ ).

\section{Total serum peroxides}

Serum peroxide concentrations were determined by commercially available rapid enzymatic assay (TOC, LND, Germany) as described before (Tatzber et al. 2003). The basis of the assay is the reaction of peroxidase with serum peroxides using tetra-methyl-benzidine (TMB, Sigma, USA) as the chromogenic substrate. Results were expressed as equivalents of $\mathrm{H}_{2} \mathrm{O}_{2}$ (in $\mu \mathrm{M}$ ).

\section{Statistical analysis}

Results are presented as a mean \pm standard error. The differences between mean values were accepted as significant when the error probability was less than $5 \%(p<0.05)$ using the Student's $t$-test. Correlation between different parameters of oxidative stress and the anaerobic threshold was calculated by using the Spearman correlation. For the statistical evaluation we used SigmaStat 3.0 for Windows (Jandel Corporation, San Rafael, CA, USA) program.

\section{Results}

Exercise induced ${ }^{\circ} \mathrm{OH}$ generation (2,3-DHB amounts) on the average by $37.5 \%$ after ergometry ( $p<0.05$, Fig. 1$)$. Total serum peroxides did not significantly change, although a tendency of decreased levels was noticed after the exercise (average decrease by $17.5 \%$ ). Total serum antioxidant capacity was higher by $39.7 \%$ after the exercise $(p<0.05)$, which was paralleled with the increase in the ${ }^{\circ} \mathrm{OH}$ radical production after ergometry (Fig. 1).

To evaluate the change of redox status before and after exercise, correlations were calculated with the AT as a parameter of physical performance, as well as between each method to determine the oxidative stress response of the healthy subjects (Table 2). The AT has shown negative correlation with the ${ }^{\circ} \mathrm{OH}$ radical production $(r=-0.92)$ and with the total serum peroxides $(r=-0.75)$. On the other hand, the rise in total antioxidant capacity after exercise showed positive correlation $(r=0.81)$ with the AT. Therefore, better physical condition resulted in lower production of ${ }^{\circ} \mathrm{OH}$ radical and in more pronounced increase of the antioxidant capacity.

The three methods for determination of oxidative parameters in the blood were also evaluated by analyzing correlation between each other (Table 2). The correlation of 'OH radical formation and total serum peroxides did not show prominent correlation $(r=0.24)$. On the other hand, correlation between ${ }^{\circ} \mathrm{OH}$ radical production and total antioxidant capacity of serum showed negative correlation $(r=$ $-0.51)$. Similarly, total peroxide level and total antioxidant capacity of serum were also in negative correlation $(r=$ $-0.51)$. 


\section{-OH radical formation}

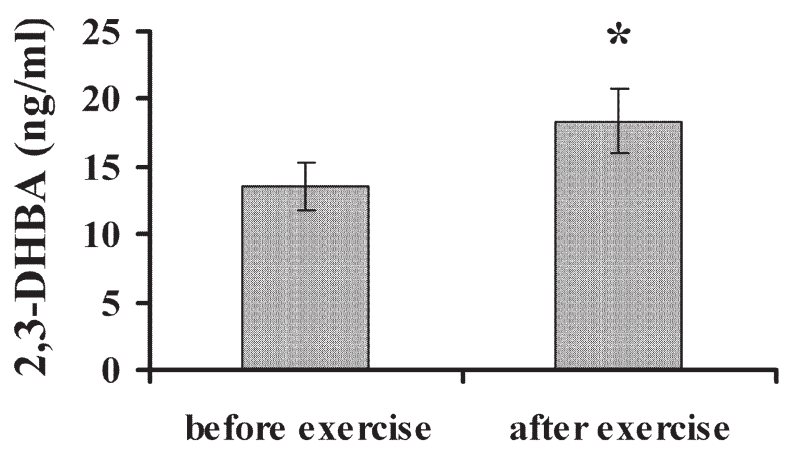

Total peroxides

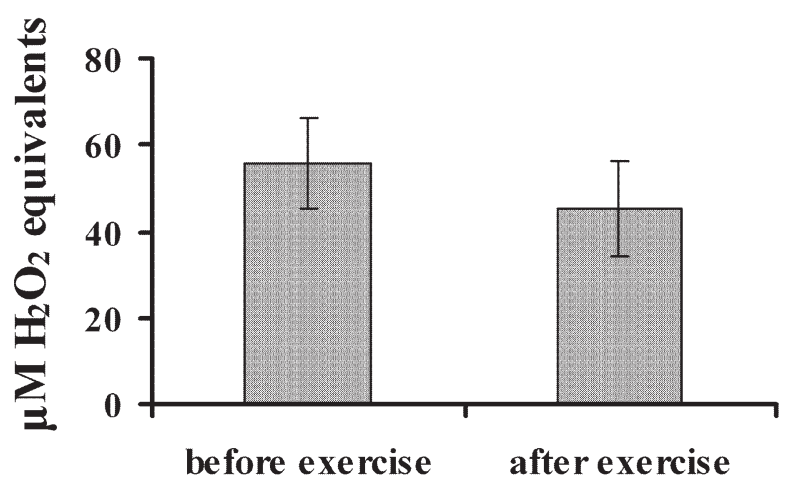

Total antioxidant capacity

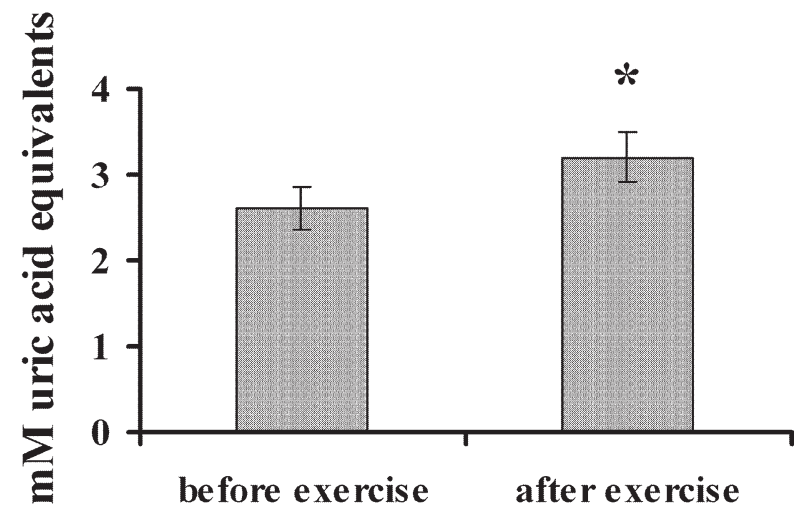

Fig. 1. Oxidative stress response of healthy male volunteers to ergometry. Redox status was evaluated by measuring plasma level of hydroxyl radical, total serum peroxides, and total serum antioxidant capacity.

*significant difference $(p<0.05)$ compared to values before exercise.

\section{Discussion}

The results obtained show that physical exercise induces ${ }^{\circ} \mathrm{OH}$ radical production and consequent oxidative stress response. This is in consistence with other recent findings showing that exhaustive exercise induces oxidative
Table 2. Correlations of physical condition and redox status biomarkers. Physical condition, measured by the speed at the anaerobic threshold (AT), 2,3-DHBA concentration and total serum peroxides (TOC) were correlated to TOC and total antioxidant capacity (TAC) in blood measured before and after exercise.

\begin{tabular}{lccc}
\hline & 2,3 DHBA & TOC & TAC \\
\hline AT & -0.92 & -0.75 & 0.81 \\
2,3DHBA & - & 0.24 & -0.51 \\
TOC & - & - & -0.51 \\
\hline
\end{tabular}

stress response (Gomez-Cabrera et al. 2008). However, while previous studies done on healthy human subjects found that exercise increased ROS production in skeletal muscles (Liu et al. 1999; Mastaloudis et al. 2001), our results show that exercise also increases systematic production of the most harmful ROS, the ${ }^{\circ} \mathrm{OH}$ radical, as evidenced by $37.5 \%$ increase of 2,3-DHBA, a specific product of nonenzymatic reaction of ${ }^{\circ} \mathrm{OH}$ radical with salicylic acid (Liu et al. 1997). Salicylic acid is a potent antioxidant, which traps the short living ${ }^{\circ} \mathrm{OH}$ radical, thereby affecting the host antioxidative mechanisms. Hence, the samples obtained after salicylic acid consumption could not be analyzed for total antioxidant capacity. Thus, the requirement of salicylic acid is major pitfall of the ${ }^{\circ} \mathrm{OH}$ radical trapping method used in our study, which also requires a relatively expensive HPLC system and the demands of tedious work of experienced researcher. Despite the inconvenience for the routine work, this method can still be considered as useful, because electron spin resonance (ESR), another efficient method for the determination of radicals, is even more difficult. ESR is frequently used for in vitro and ex vivo analyses, but not so much for in vivo studies, due to high costs and toxicity of reagents (spin traps) needed to trap the radicals. Under in vitro or ex vivo circumstances, both methods give very good correlation, although endogenous nitric oxide production might affect results of the ESR method, but not results of hydroxyl radical trapping with HPLC. Application of acetyl-salicylic acid could be associated with undesirable side effects, which do not allow application of this analytical method for some clinical studies, but it is still more convenient than the use of spin traps in humans. However, due to their sensitivity, both of these methods could be at the moment considered as gold standards to determine oxygen free radicals produced in a living system.

Hydroxyl radical could react with serum antioxidants as well as with blood peroxides yielding water and peroxyl radical. Accordingly, total serum peroxides could show a decrease in case of increased ${ }^{\circ} \mathrm{OH}$ radical, as was observed in our study. Such a decrease in total serum peroxides is in accordance with recent literature describing oxidative stress in professional sportsmen (Schippinger et al. 2009). Peroxyl radical, which might be generated upon the attack of ${ }^{\circ} \mathrm{OH}$ radical on serum peroxides, is more stable than ${ }^{\circ} \mathrm{OH}$ 
radical; hence its formation can further mobilize antioxidative mechanisms. Peroxyl radical may thus induce the increase of glutathione, glutathione peroxidases and superoxide dismutase (Parise et al. 2005), resulting in an eventual increase of total antioxidant capacity, as determined in our volunteers after exercise.

In our study, total peroxide assay did not appear as sensitive enough for determination of systematic oxidative stress. This result is opposite to the usefulness of this assay for evaluation of persistent oxidative stress during convalescence of patients with myocardial infarction (NikolicHeitzler et al. 2006). Another study showed that pre-operative delivery of a vitamin cocktail could diminish oxidative stress after vascular surgery (Wonisch et al. 2005). Therefore, it is possible that oxidative stress initially causes a decrease in available antioxidants, followed by consequent mobilization of the entire antioxidant defense and the rise of antioxidants, which may decrease the peroxide levels. In case that the overall antioxidant resources are too low, as in case of persistent oxidative stress in diseases associated with oxidative stress especially if associated with additional stress, such as surgery or inflammation, peroxides would be produced in excess. Such deteriorating situation might be avoided, as indicated by possible prevention of oxidative stress after surgery in patients with peripheral arterial occlusive disease by the use of ascorbic acid and alpha-tocopherol (Wonisch et al. 2005).

Our results are consistent in part with published results showing that total antioxidant capacity increased in the course of a competition season in alpine ski racers (Schippinger et al. 2009). In these sportsmen there were no differences in the levels of systemic antioxidants: ascorbate, tocopherols and $\beta$-carotene before and after the race, but there was a significant increase of the uric acid (potent metabolic antioxidant) blood levels after the race. Taking these facts into account, the increase in total antioxidant capacity after exercise observed in our study could be explained by a possible increase in uric acid and/or endogenous peroxidases, as oxidative stress response and countermeasure against the generation of ROS indicating an adaptive response to exercise induced oxidative stress, as manifested by the increase of the total antioxidant capacity. Our study has indeed shown that total serum antioxidant capacity was reliable for the analysis of oxidative stress response, and was in strong correlation with the physical condition of the subjects as well as with the hydroxyl radical measurement. Therefore, it is convenient for the routine usage as simple method for determination of oxidative status in healthy human subjects with physical exercise.

It is also important to emphasize good relation of the method of the ${ }^{\circ} \mathrm{OH}$ radical trapping with acetyl-salicylic acid and two relatively simple and affordable oxidative stress biomarkers, total serum peroxides and total serum antioxidative capacity. All three tests used showed good correlation with physical condition of subjects indicating that oxidative homeostasis plays important role in hormesis.
These findings suggest a possible use of exercise to induce physiological oxidative stress maintaining oxidative homeostasis. Exercise increases the overall health of the normal organism by increasing endogenous antioxidant mechanisms with consequential increase in resistance to the pathology of severe oxidative stress. However, this should be done carefully, without the use of additional antioxidants that could replace the response of endogenous antioxidants to exercise (Vina et al. 2000). If done optimally, exercise could reflect the health status and also could support convalescence in both acute and chronic oxidative stress associated disorders (Svacinová et al. 2008). Finally, it should be mentioned that comparison of the three assays used has validated ergometry as a valuable tool to induce systemic oxidative stress response, which should be further studied in various patients, in particular those suffering from diseases associated with oxidative stress.

\section{Conclusions}

The results of the study lead to the following conclusions:

a. Exercise induces hydroxyl radical production

b. The induced oxidative stress stimulates endogenous antioxidant systems, thereby increasing the total antioxidant capacity

c. Such a stress response may lead to a marginal decrease of total peroxides

d. All these biomarkers of systemic oxidative stress correlate strongly with the physical performance of the healthy volunteers

e. A combination of different oxidative stress biomarkers is necessary to determine the interaction between the complex oxidant/antioxidant network of systemic oxidative stress response and oxidative homeostasis

\section{Acknowledgment}

This study was supported by Croatian Ministry of Science, Education and Sports and by European Commission Grant COST Action B35. The authors wish to thank the editors and the reviewers of the manuscript for very stimulating and constructive comments.

\section{References}

Ates, O., Azizi, S., Alp, H.H., Kiziltunc, A., Beydemir, S., Cinici, E., Kocer, I. \& Baykal, O. (2009) Decreased serum paraoxonase 1 activity and increased serum homocysteine and malondialdehyde levels in age-related macular degeneration. Tohoku J. Exp. Med., 217, 17-22.

Bejma, J. \& Ji, L.L. (1999) Aging and acute exercise enhance free radical generation in rat skeletal muscle. J. Appl. Physiol., 87, 465-470.

Bloomer, R.J. (2008) Effect of exercise on oxidative stress biomarkers. Adv. Clin. Chem., 46, 1-50.

Borovic, S., Meinitzer, A., Loncaric, I., Sabolovic, S., Wildburger, R., Tillian, M., Martinac, P., Stipancic, I. \& Zarkovic, N. (2000) Monitoring influence of surgical stress on formation of hydroxyl radicals in tumor bearing rats by measuring salicylic acid metabolites. Electron. J. Int. Fed. Clin. Chem. Lab. Med., $12,1-4$. 
Braughler, J.M. \& Hall, E.D. (1989) Central nervous system trauma and stroke: I. Biochemical considerations for oxygen radical formation and lipid peroxidation. Free Radic. Biol. Med., 6, 289-301.

Cindric, M., Cipak, A., Mrakovcic, L., Zarkovic, K., Borovic, S., Wildburger, R. \& Zarkovic, N. (2009) Homeostasis, stress and aging. Turk. Klin. Tip. Bilim Der., 29, s3-s5.

Esterbauer, H., Schaur, R.J. \& Zollner, H. (1991) Chemistry and biochemistry of 4-hydroxynonenal, malondialdehyde, and related aldehydes. Free Radic. Biol. Med., 11, 81-128.

Gomez-Cabrera, M.C., Domenech, E., Ji, L.L. \& Vina, J. (2006) Exercise as an antioxidant: it up-regulates important enzymes for cell adaptations to exercise. Sci. Sports, 21, 85-89.

Gomez-Cabrera, M.C., Domenech, E. \& Vina, J. (2008) Moderate exercise is an antioxidant: Upregulation of antioxidant genes by training. Free Radic. Biol. Med., 44, 126-131.

Hall, E.D. \& Braughler, J.M. (1989) Central nervous system trauma and stroke: II. Physiological and pharmacological evidence for involvement of oxygen radicals and lipid peroxidation. Free Radic. Biol. Med., 6, 303-313.

Halliwell, B. (1996) Antioxidants in human health and disease. Annu. Rev. Nutr., 16, 33-50.

Hellsten-Westing, Y. (1993) Immunohistochemical localization of xanthine oxidase in human cardiac and skeletal muscle. Histochemistry, 100, 215-222.

Isik, A., Koca, S.S., Ustundag, B. \& Selek, S. (2007) Decreased total antioxidant response and increased oxidative stress in Behcet's disease. Tohoku J. Exp. Med., 212, 133-141.

Jamieson, D.J. (1998) Oxidative responses of the yeast Saccharomyces cerevisiae. Yeast, 14, 1511-1527.

Ji, L.L., Radak, Z. \& Goto, S. (2008) Hormesis and exercise: How the cell copes with oxidative stress. Am. J. Pharmacol. Toxicol., 3, 44-58.

Khoschsorur, G.A., Winklhofer-Roob, B.M., Rabl, H., Auer, Th., Peng, Z. \& Schaur, R.J. (2000) Evaluation of a sensitive HPLC method for the determination of malondialdehyde, and application of the method to different biological materials. Chromatographia, 52, 181-184.

Liu, L., Leech, J.A., Urch, R.B. \& Silverman, F.S. (1997) In vivo salicylate hydroxylation: a potential biomarker for assessing acute ozone exposure and effects in humans. Am. J. Respir. Crit. Care Med., 156, 1405-1412.

Liu, M.L., Bergholm, R., Makimattila, S., Lahdenpera, S., Valkonen, M., Hilden, H., Yki-Jarvinen, H. \& Taskinen, M.R. (1999) A marathon run increases the susceptibility of LDL to oxidation in vitro and modifies plasma antioxidants. Am. J. Physiol., 276, E1083-E1091.

Mamiya, T., Katsuoka, F., Hirayama, A., Nakajima, O., Kobayashi, A., Maher, J.M., Matsui, H., Hyodo, I., Yamamoto, M. \& Hosoya, T. (2008) Hepatocyte-specific deletion of heme oxygenase- 1 disrupts redox homeostasis in basal and oxidative environments. Tohoku J. Exp. Med., 216, 331-339.

Mastaloudis, A., Leonard, S.W. \& Traber, M.G. (2001) Oxidative stress in athletes during extreme endurance exercise. Free Radic. Biol. Med., 31, 911-922.

McCabe, D.R., Maher, T.J. \& Acworth, I.N. (1997) Improved method for the estimation of hydroxyl free radical levels in vivo based on liquid chromatography with electrochemical detection. J. Chromatogr. B Biomed. Sci. Appl., 691, 23-32.

Meyer, T., Lucia, A., Earnest, C.P. \& Kindermann, W. (2005) A conceptual framework for performance diagnosis and training prescription from submaximal gas exchange parameters - theory and application. Int. J. Sports Med., 26, S38-48.

Nikolic-Heitzler, V., Rabuzin, F., Tatzber, F., Vrkic, N., Bulj, N., Borovic, S., Wonisch, W., Sunko, B.M. \& Zarkovic, N. (2006) Persistent oxidative stress after myocardial infarction treated by percutaneous coronary intervention. Tohoku J. Exp. Med., 210, 247-255.

Parise, G., Phillips, S.M., Kaczor, J.J. \& Tarnopolsky, M.A. (2005) Antioxidant enzyme activity is up-regulated after unilateral resistance exercise training in older adults. Free Radic. Biol. Med., 39, 289-295.

Schippinger, G., Fankhauser, F., Abuja, P.M., Winklhofer-Roob, B.M., Nadlinger, K., Halwachs-Baumann, G. \& Wonisch, W. (2009) Competitive and seasonal oxidative stress in elite alpine ski racers. Scand. J. Med. Sci. Sports, 19, 206-212.

Svacinová, H., Nováková, M., Placheta, Z., Kohzuki, M., Nagasaka, M., Minami, N., Dobšák, P. \& Siegelová, J. (2008) Benefit of combined cardiac rehabilitation on exercise capacity and cardiovascular parameters in patients with type 2 diabetes. Tohoku J. Exp. Med., 215, 103-111.

Tasoulis, M.K., Livaditi, O., Stamatakos, M., Stefanaki, C., Paneris, P., Prigouris, P., Flevari, A., Goutas, N., Vlachodimitropoulos, D., Villiotou, V. \& Douzinas, E.E. (2009) High concentrations of reactive oxygen species in the BAL fluid are correlated with lung injury in rabbits after hemorrhagic shock and resuscitation. Tohoku J Exp Med., 219, 193-199.

Tatzber, F., Griebenow, S., Wonisch, W. \& Winkler, R. (2003) Dual method for the determination of peroxidase activity and total peroxides-iodide leads to a significant increase of peroxidase activity in human sera. Anal. Biochem., 316, 147-153.

Urso, M.L. \& Clarkson, PM. (2003) Oxidative stress, exercise, and antioxidant supplementation. Toxicology, 189, 41-54.

Vina, J., Gomez-Cabrera, M.C., Lloret, A., Marquez, R., Minana, J.B., Pallardo, F.V. \& Sastre, J. (2000) Free radicals in exhaustive physical exercise: mechanism of production, and protection by antioxidants. IUBMB Life, 50, 271-277.

Wildburger, R., Mrakovcic, L., Stroser, M., Andrisic, L., Borovic Sunjic, S., Zarkovic, K. \& Zarkovic, N. (2009) Lipid Peroxidation and Age-Associated Diseases-Cause or Consequence? Turk. Klin. Tip. Bilim Der., 29, 189-193.

Wonisch, W., Uhl, K., Schimetta, W. \& Rabl, H. (2005) Pre-operative delivery of a vitamin cocktail diminished oxidative stress after vascular surgery in PAD patients-a pilot investigation. Biofactors, 24, 299-303.

Zarkovic, N. (2003) 4-Hydroxynonenal as a bioactive marker of pathopysiological processes. Mol. Aspects Med., 24, 281-291.

Zarkovic, N. (2009) Protein-aldehydic adducts as biomarkers of oxidative stress, lipid peroxidation and oxidative homeostasis. Free Radic. Res., 43, 33. 